Canadian

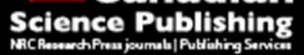

Canadian Journal of Physics

Revue canadienne de physique

Bound States of Atomic Josephson Vortices

\begin{tabular}{|r|l|}
\hline Journal: & Canadian Journal of Physics \\
\hline Manuscript ID & cjp-2016-0599.R1 \\
\hline Manuscript Type: & Article \\
\hline Date Submitted by the Author: & 28 -Oct-2016 \\
\hline Complete List of Authors: & $\begin{array}{l}\text { Qadir, Muhammad; University of Engineering \& Technology Lahore, } \\
\text { Mathematics; } \\
\text { Tahir, Usama; University of Engineering \& Technology Lahore, Mathematics }\end{array}$ \\
\hline Keyword: & $\begin{array}{l}\text { Bose Einstein Condensation, Bose-Josephson junction, Josephson vortices, } \\
\text { Dark solitons, Stability }\end{array}$ \\
\hline
\end{tabular}




\title{
Bound States of Atomic Josephson Vortices
}

\author{
Muhammad Irfan Qadir and Usama Tahir \\ Department of Mathematics, University of Engineering \& Technology, \\ Lahore 54890, Pakistan
}

Corresponding author: Muhammad Irfan Qadir; Email: mirfan@uet.edu.pk

PACS: 03.75.Lm, 03.75.Mn, 74.50.+r.

\begin{abstract}
We study the existence and stability of the bound state Josephson vortices solution in two parallel quasi one-dimensional coupled Bose-Einstein condensates. The system can be elucidated by linearly coupled GrossPitaevskii equations. The purpose of this study is to investigate the effects of altering the strength of coupling between the two condensates over the stability of the bound state Josephson vortices. It is found that the stability of bound state Josephson vortices depends on the value of coupling strength. However, at a critical value of coupling parameter, the Josephson vortices solution transforms into a coupled dark soliton.
\end{abstract}

Keywords: Bose-Einstein Condensation, Bose-Josephson junction, Josephson vortices, Dark solitons, Stability.

\section{INTRODUCTION}

Bose-Einstein condensate (BEC) is a quantum state of matter in which atoms of weakly interacting alkali metals at very low temperature undergo into a single collective quantum state $[1,2,3]$. Bose-Einstein condensation was first predicted by Indian physicist Satyendra Nath Bose in 1924 and then developed by Albert Einstein in 1925. The experimental realization in 1995 and great progress in the theoretical studies of BEC of weakly interacting atoms of alkali metals $[4,5,6]$ encourages the investigation of non-linear characteristics of matter waves. The phenomena of BEC leads to the study of different excited states such as solitons [7, 8] and vortices [9].

In BEC and non-linear optics, solitons are self-reinforcing wave packets caused by the dispersion and non-linearity [10]. There exist different configurations of solitons but the most studied configurations are bright and dark solitons for attractive and repulsive Bose gases respectively.

Dark solitons also known as the "kink states" is a captivating subject for study. The stability of dark solitons is dependent on the geometry and non-linearity of the channel [11]. In one dimension the radial 
motion of the dark solitons is strongly restricted in a cylindrical trap and they go through zero point radial oscillations. As a result the snake deformation is suppressed and dark solitons become stable [12]. However, the dark solitons are unstable in more than one dimensions because their energies are always higher than the ground state of BEC [13].

Topological configurations such as vortices are caused due to snake deformation in dark solitons. Around a one dimensional core where density vanishes vortices have quantized flow in the medium of quantum fluid. Vortices have been observed in superfluid helium, superconductors and in BEC [9].

A paradigm of quantum mechanics that generally occurs on a nanoscopic scale is the tunneling through a barrier. Smerzi and his colleagues $[14,15,16]$ introduced the idea of tunneling of atoms of BEC that was analogues to the electron tunneling between two superconductors joined together through a flimsy insulator. Such tunneling for an array of short Josephson junctions [17] and in a single Bose- Josephson junction [18] was observed experimentally. Kaurov and Kuklov extended the idea of small BoseJosephson junction to long Bose-Josephson junction [19, 20]. The notion of long Bose-Josephson junction was similar to that of long superconducting Josephson junction. They showed [19] that in two weakly coupled waveguides of BEC, the atomic vortices could be seen. These atomic vortices called Josephson vortices are alike the Josephson fluxon in long superconducting Josephson junction. Furthermore, the Josephson vortices can be transformed into dark solitons and vice versa by having a control on the coupling strength between the two condensates.

As concerns various effects in the model of the BEC trapped in tunnel-coupled parallel potential pipes, the existence and stability of the Josephson vortices and their conversion into dark solitons were studied in $[21,22]$. Numerical stability analysis showing the Josephson tunneling of dark solitons in a double well potential was investigated in [23]. The stability of the basis functions used to describe the dynamics of bosons in the two wells of an external trap was presented in [24].

In this work, we study the existence and stability of bound states of Josephson vortices in two one dimensional coupled BEC. Typically, we analyze the effects of variation in the value of coupling strength on the stability of bound states of Josephson vortices in a coupled BEC.

The paper is formatted as follows. In section 2, we consider the coupled system of nonlinear GrossPitaevskii equations that describes BEC and find the bound states of Josephson vortices numerically. In section 3, we discuss the linear stability of the bound state of Josephson vortices while changing the value of coupling parameter. We conclude our results in section 4. 


\section{MATHEMATICAL MODEL AND DESCRIPTION}

We begin by considering a set of two parallel coupled effectively one dimensional cigar-shaped BEC. The intra atomic interaction is supposed to be repulsive. The dynamics of quantum fields known as bosonic fields is governed by the integral

$$
\int L d x d t
$$

where $t$ represents the time and $x$ the space variable. $L$ is the Lagrangian density that comprises of three parts namely $L_{1}, L_{2}$ and $L_{12} . L_{1}$ and $L_{2}$ describe the dynamics of atoms in the two waveguides along the $x$ direction and are given as

$$
\begin{aligned}
& L_{1}=\frac{i\left(\overline{\chi_{1}} \frac{\partial \chi_{1}}{\partial t}+\chi_{1} \frac{\partial \overline{\chi_{1}}}{\partial t}\right)}{2}-\frac{\left|\nabla \chi_{1}\right|^{2}}{2}+\omega_{1}\left|\chi_{1}\right|^{2}-\frac{\mu\left|\chi_{1}\right|^{4}}{2} \\
& L_{2}=\frac{i\left(\overline{\chi_{2}} \frac{\partial \chi_{2}}{\partial t}+\chi_{2} \frac{\partial \overline{\chi_{2}}}{\partial t}\right)}{2}-\frac{\left|\nabla \chi_{2}\right|^{2}}{2}+\omega_{2}\left|\chi_{2}\right|^{2}-\frac{\mu\left|\chi_{2}\right|^{4}}{2},
\end{aligned}
$$

while $L_{12}$ represents the Josephson tunneling between the condensates and is given as

$$
L_{12}=k\left(\overline{\chi_{1}} \chi_{2}+\chi_{1} \overline{\chi_{2}}\right) \text {. }
$$

Here $\chi_{1}$ and $\chi_{2}$ denotes the bosonic fields and bar represents the complex conjugate. $k$ is the coupling parameter and $\omega_{1}=\omega_{2}=\omega$ denotes the chemical potential that is assumed to be same in the two waveguides. $\mu>0$ is the nonlinearity coefficient and $i=\sqrt{-1}$. Employing equations (1-4) and using the variational principle yields

$$
\begin{aligned}
& i \frac{\partial \chi_{1}}{\partial t}=-\frac{1}{2} \frac{\partial^{2} \chi_{1}}{\partial x^{2}}+\mu\left|\chi_{1}\right|^{2} \chi_{1}-\omega \chi_{1}-k \chi_{2} \\
& i \frac{\partial \chi_{2}}{\partial t}=-\frac{1}{2} \frac{\partial^{2} \chi_{2}}{\partial x^{2}}+\mu\left|\chi_{2}\right|^{2} \chi_{2}-\omega \chi_{2}-k \chi_{1} .
\end{aligned}
$$

For seeking the steady state solutions, we substitute $\frac{\partial \chi_{1}}{\partial t}=0=\frac{\partial \chi_{2}}{\partial t}$ in equations (5) and (6) and obtain

$$
\begin{aligned}
& \frac{1}{2} \frac{\partial^{2} \chi_{1}}{\partial x^{2}}-\mu\left|\chi_{1}\right|^{2} \chi_{1}+\omega \chi_{1}+k \chi_{2}=0 \\
& \frac{1}{2} \frac{\partial^{2} \chi_{2}}{\partial x^{2}}-\mu\left|\chi_{2}\right|^{2} \chi_{2}+\omega \chi_{2}+k \chi_{1}=0
\end{aligned}
$$

Since $\chi_{1}$ and $\chi_{2}$ are complex, we can write $\chi_{1}=u_{1}+i v_{1}$ and $\chi_{2}=u_{2}+i v_{2}$ in equations (7) and (8). Then after equating the real and imaginary parts on both sides, we obtain the following equations 


$$
\begin{aligned}
& \frac{1}{2} \frac{\partial^{2} u_{1}}{\partial x^{2}}-\mu\left(u_{1}^{3}+u_{1} v_{1}^{2}\right)+\omega u_{1}+k u_{2}=0 \\
& \frac{1}{2} \frac{\partial^{2} v_{1}}{\partial x^{2}}-\mu\left(v_{1}^{3}+u_{1}{ }^{2} v_{1}\right)+\omega v_{1}+k v_{2}=0 \\
& \frac{1}{2} \frac{\partial^{2} u_{2}}{\partial x^{2}}-\mu\left(u_{2}^{3}+u_{2} v_{2}^{2}\right)+\omega u_{2}+k u_{1}=0 \\
& \frac{1}{2} \frac{\partial^{2} v_{2}}{\partial x^{2}}-\mu\left(v_{2}{ }^{3}+u_{2}{ }^{2} v_{2}\right)+\omega v_{2}+k v_{1}=0
\end{aligned}
$$

We discretize equations (9), (10), (11) and (12) to obtain a system of nonlinear algebraic equations. Solving this system of equations numerically using Neumann boundary conditions, we obtain the bound state Josephson vortices solution which is shown in Fig. (1). In the settings of parametrically driven nonlinear Schrodinger equation, the stability of the bound state of Josephson vortices was studied in [25, 26] where these states correlate with the Bloch-Bloch states.

\section{STABILITY OF BOUND STATE SOLUTION}

For studying the stability of bound state solution, we first suppose that $\chi_{1}^{*}$ and $\chi_{2}^{*}$ are the time independent solutions of system of equations (5) and (6). We now perturb these solutions by giving very small perturbations $\varepsilon_{1}(\mathrm{x}, \mathrm{t})$ and $\varepsilon_{2}(\mathrm{x}, \mathrm{t})$ in these solutions $\chi_{1}^{*}$ and $\chi_{2}^{*}$ respectively, i.e.

$$
\begin{aligned}
& \chi_{1}(x, t)=\chi_{1}^{*}(x)+\varepsilon_{1}(x, t), \\
& \chi_{2}(x, t)=\chi_{2}^{*}(x)+\varepsilon_{2}(x, t) .
\end{aligned}
$$

Substituting the values of $\chi_{1}$ and $\chi_{2}$ from equations (13) and (14) into equations (5) and (6) and then linearizing the resulting equations yields

$$
\begin{aligned}
& i \frac{\partial \varepsilon_{1}}{\partial t}=-\frac{1}{2} \frac{\partial^{2} \varepsilon_{1}}{\partial x^{2}}+\mu\left(\chi_{1}{ }^{*}\right)^{2} \overline{\varepsilon_{1}}+2 \mu\left|\chi_{1}{ }^{*}\right|^{2} \varepsilon_{1}-\omega \varepsilon_{1}-k \varepsilon_{2}, \\
& i \frac{\partial \varepsilon_{2}}{\partial t}=-\frac{1}{2} \frac{\partial^{2} \varepsilon_{2}}{\partial x^{2}}+\mu\left(\chi_{2}{ }^{*}\right)^{2} \overline{\varepsilon_{2}}+2 \mu\left|\chi_{2}{ }^{*}\right|^{2} \varepsilon_{2}-\omega \varepsilon_{2}-k \varepsilon_{1} .
\end{aligned}
$$

Taking complex conjugate of equations (15) and (16) and substituting $\varepsilon_{1}=\alpha_{1}, \overline{\varepsilon_{1}}=\beta_{1}, \varepsilon_{2}=\alpha_{2}$, $\overline{\varepsilon_{2}}=\beta_{2}$, we obtain

$$
i \frac{\partial \alpha_{1}}{\partial t}=-\frac{1}{2} \frac{\partial^{2} \alpha_{1}}{\partial x^{2}}+\mu\left(\chi_{1}^{*}\right)^{2} \beta_{1}+2 \mu\left|\chi_{1}{ }^{*}\right|^{2} \alpha_{1}-\omega \alpha_{1}-k \alpha_{2}=\sigma \alpha_{1}
$$




$$
\begin{aligned}
& i \frac{\partial \alpha_{2}}{\partial t}=-\frac{1}{2} \frac{\partial^{2} \alpha_{2}}{\partial x^{2}}+\mu\left(\chi_{2}{ }^{*}\right)^{2} \beta_{2}+2 \mu\left|\chi_{2}{ }^{*}\right|^{2} \alpha_{2}-\omega \alpha_{2}-k \alpha_{1}=\sigma \alpha_{2} \\
& i \frac{\partial \beta_{1}}{\partial t}=\frac{1}{2} \frac{\partial^{2} \beta_{1}}{\partial x^{2}}-\mu\left(\overline{\chi_{1}{ }^{*}}\right)^{2} \alpha_{1}-2 \mu\left|\chi_{1}{ }^{*}\right|^{2} \beta_{1}+\omega \beta_{1}+k \beta_{2}=\sigma \beta_{1} \\
& i \frac{\partial \beta_{2}}{\partial t}=\frac{1}{2} \frac{\partial^{2} \beta_{2}}{\partial x^{2}}-\mu\left(\overline{\chi_{2}{ }^{*}}\right)^{2} \alpha_{2}-2 \mu\left|\chi_{2}{ }^{*}\right|^{2} \beta_{2}+\omega \beta_{2}+k \beta_{1}=\sigma \beta_{2} .
\end{aligned}
$$

The above system of equations (17), (18), (19) and (20) represents an eigenvalue problem $\mathrm{A} Y=\sigma Y$ with the eigenvalue $\sigma$ and the corresponding eigenvector $Y$. Discretizing the above equations and applying the same boundary conditions used before, one can write the coefficient matrix A as

$$
\mathrm{A}=\left[\begin{array}{cccc}
L_{1} & -C & M_{1} & 0 \\
-C & L_{2} & 0 & M_{2} \\
-\overline{M_{1}} & 0 & -L_{1} & C \\
0 & -\overline{M_{2}} & C & -L_{2}
\end{array}\right]
$$

where

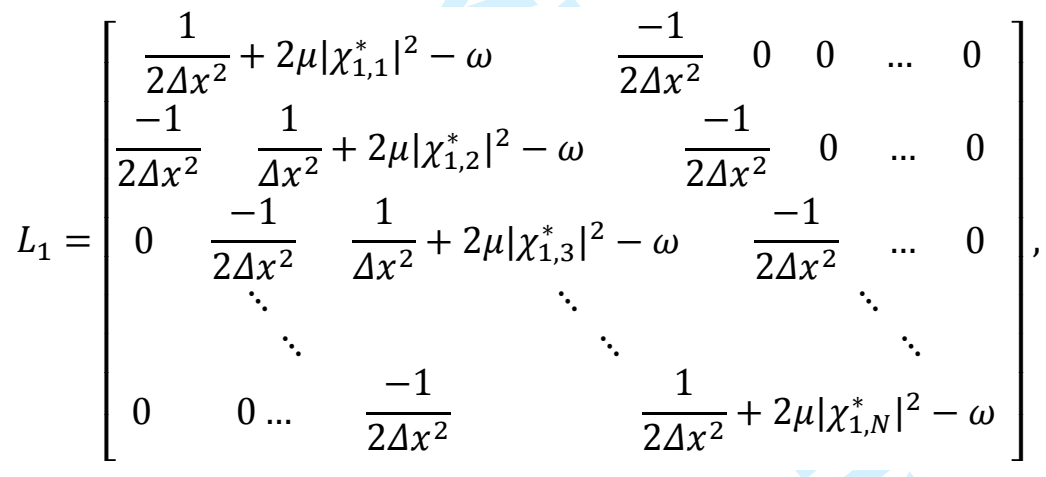

$$
\begin{aligned}
& L_{2}=\left[\begin{array}{ccccccc}
\frac{1}{2 \Delta x^{2}}+2 \mu\left|\chi_{2,1}^{*}\right|^{2}-\omega & \frac{-1}{2 \Delta x^{2}} & 0 & 0 & \ldots & 0 \\
\frac{-1}{2 \Delta x^{2}} & \frac{1}{\Delta x^{2}}+2 \mu\left|\chi_{2,2}^{*}\right|^{2}-\omega & \frac{-1}{2 \Delta x^{2}} & 0 & \ldots & 0 \\
0 & \frac{-1}{2 \Delta x^{2}} & \frac{1}{\Delta x^{2}}+2 \mu\left|\chi_{2,3}^{*}\right|^{2}-\omega & \frac{-1}{2 \Delta x^{2}} & \ldots & 0 \\
& \ddots & \ddots & & & \ddots & \\
& 0 \ldots & \frac{-1}{2 \Delta x^{2}} & & & \frac{1}{2 \Delta x^{2}}+2 \mu\left|\chi_{2, N}^{*}\right|^{2}-\omega
\end{array}\right],
\end{aligned}
$$




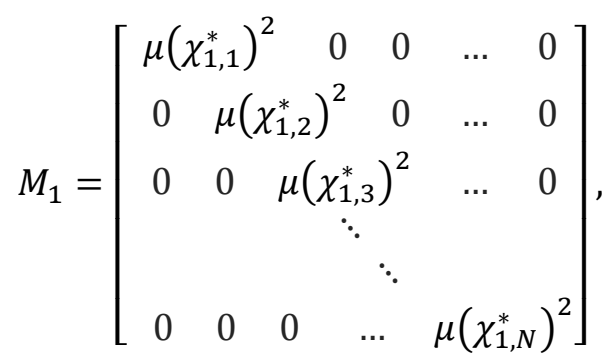

$$
\begin{aligned}
& M_{2}=\left[\begin{array}{ccccc}
\mu\left(\chi_{2,1}^{*}\right)^{2} & 0 & 0 & \ldots & 0 \\
0 & \mu\left(\chi_{2,2}^{*}\right)^{2} & 0 & \ldots & 0 \\
0 & 0 & \mu\left(\chi_{2,3}^{*}\right)^{2} & \ldots & 0 \\
& & \ddots & & \\
& & & \ddots & \\
0 & 0 & 0 & \ldots & \mu\left(\chi_{2, N}^{*}\right)^{2}
\end{array}\right] \text {, } \\
& C=\left[\begin{array}{ccccc}
k & 0 & 0 & \ldots & 0 \\
0 & k & 0 & \ldots & 0 \\
0 & 0 & k & \ldots & 0 \\
& & & \ddots & \\
& & & \ddots & \\
0 & 0 & 0 & \ldots & k
\end{array}\right] .
\end{aligned}
$$

For the stability of the bound state solution, all eigenvalues should be real. Nonetheless, the solution will become unstable if atleast one of the eigenvalue is complex.

The eigenvalues of the stability matrix A are found and are depicted in Fig. 2. One can find that most of the eigenvalues have their imaginary parts zero and as such they are lying on the horizontal axis. However, two pairs of eigenvalues are complex and are shown in the complex plane. Due to these two pairs of eigenvalues, the bound state Josephson vortices solution is prone to instability.

We then investigate the stability of the solution for different values of coupling strength $k$. In Fig. 3 , the red dotted curve is the stability curve for the bound state Josephson vortices solution. This curve indicates that the bound state solution is stable for $k \leq 0.2$. For $k>0.2$, a pair of eigenvalues emerges from the spectrum of those eigenvalues that are lying horizontally. Due to this pair of eigenvalues which is lying vertically, the solution becomes unstable. The instability persists in the interval $0.2<k<0.26$ and ultimately the solution becomes stable for $k \geq 0.26$. However, the bound state solution transforms into a coupled dark soliton at $k=1 / 3$. The coupled dark soliton was found to be unstable for $k<1 / 3$ but becomes stable for $k \geq 1 / 3$. This agrees with the result in [19] as depicted in Fig. (3) by green solid curve.

To verify the above results, the system of equations (5) and (6) can be solved using any appropriate numerical technique. In particular, we apply Runge-Kutta method with fourth order accuracy and use the bound state Josephson vortices solution as the initial condition. The contour plot for the time evolution of 
bound state Josephson vortices solution is shown in Fig.4. The emergence of radiation at approximately $t=300$ indicates the instability of the solution and justifies the results already obtained. The instability causes the solution to move away from the mean position.

\section{CONCLUDING REMARKS}

In this paper, we have examined the existence and stability of bound state Josephson vortices solution in two quasi one-dimensional parallel coupled BEC. Our analysis is based on a coupled system of GrossPitaevskii equations. It has been found that the bound state Josephson vortices solution exists for $0<k<$ $1 / 3$. The stability of bound state Josephson vortices solution has been investigated while varying the value of coupling parameter. The solution was found to be unstable in the region $0.2<k<0.26$, while it is stable in its remaining domain of existence. At $k=1 / 3$, the bound state Josephson vortices solution transforms into a coupled dark soliton solution. The coupled dark soliton solution exists and remain unstable for $k<1 / 3$ and becomes stable for $k \geq 1 / 3$.

\section{REFERENCES}

[1] C. J. Pethick and H. Smith. Bose-Einstein Condensation in Dilute Gases. Cambridge University Press, UK (2001).

[2] A. Griffin, D. W. Snoke and S. Stringari. Bose-Einstein Condensation. Cambridge University Press, UK (1995).

[3] L. P. Pitaevskii and S. Stringari, Bose-Einstein Condensation and Superfluidity, Cambridge University Press, UK (2016).

[4] M. H. Anderson, J. R. Ensher, M. R. Matthews, C. E. Wieman and E. A. Cornell, Science 269: 198-201 (1995).

[5] K. B. Davis, M. O. Mewes, M. R. Andrews, N. J. van Druten, D. S. Durfee, D. M. Kurn and W. Ketterle, Phys. Rev. Lett.. 75: 3969-3973 (1995).

[6] C. C. Bradley, C. A. Sackett and R. G. Hulet, Phys. Rev. Lett. 75: 985-989 (1997).

[7] K. E. Strecker, G. B. Partridge, A. G. Truscott and R. G. Hulet, Nature 417: 150-153 (2002).

[8] Burger, S. K. Bongs and M. Lewenstein. Phys. Rev. Lett. 83: 5198-5201 (1999).

[9] M. R. Matthews, B. P. Anderson, P. C. Haljan, D. S. Hall, C. E. Wieman and E. A. Cornell, Phys. Rev. Lett. 83, 2498-2501 (1999). 
[10] P. G. Drazin and R. S. Johnson, Solitons: An Introduction. Cambridge University Press, UK (1989).

[11] Y. S. Kivshar and B. Luther-Davies, Phys. Rep. 298, 81-197 (1998).

[12] A. E. Muryshev, H. B. Heuvell and G. V. Shlyapnikov. Phys. Rev. A 60: R2665-R2668 (1999).

[13] P. O. Fedichev, A. E. Muryshev and G. V. Shlyapnikov, Phys. Rev. A 60: 3220-3224 (1999).

[14] A. Smerzi, S. Fantoni, S. Giovanazzi and S. R. Shenoy. Phys. Rev. Lett. 79: 4950-4953 (1997).

[15] S. Raghavan, A. Smerzi, S. Fantoni and S. R. Shenoy. Phys. Rev. A 59: 620-633 (1999).

[16] S. Giovanazzi, A. Smerzi and S. Fantoni. Phys. Rev. Lett. 84: 4521-4524 (2000).

[17] F. S. Cataliotti, S. Burger, C. Fort, P. Maddaloni, F. Minardi, A. Trombettoni, A. Smerzi and M. Inguscio. Science 293: 843-846 (2001).

[18] M. Albiez, R. Gati, J. Folling, S. Hunsmann, M. Cristiani and M. K. Oberthaler. Phys. Rev. Lett. 95, 010402 (2005).

[19] V. M. Kaurov and A. B. Kuklov. Phys. Rev. A 71: 011601-011601- 4 (2005).

[20] V. M. Kaurov and A. B. Kuklov. Phys. Rev, A 73: 013627 (2006).

[21] M. I. Qadir, H. Susanto and P. C. Matthews. J. Phys.B. At., Mol. Opt. Phys. 45: 035004 (2012).

[22] M. I. Qadir and N. Irshad. Proceeding of the Pakistan Academy of Sciences, A Physical and Computational Sciences 53: 17-24 (2016).

[23] H. Susanto, J. Cuevas and P. Kruger. J. Phys.B: At., Mol. Opt. Phys. 44: 095003 (2011).

[24] M. I. Qadir and M. D. A. Rana. Proceedings of the Pakistan Academy of Sciences 52: 247-254 (2015) .

[25] I. V. Barashenkov, S. R. Woodford and E. V. Zemlyanaya. Phys. Rev. Lett. 90: 054103 (2003).

[26] I. V. Barashenkov, S.R. Woodford and E. V. Zemlyanaya. Phys. Rev. E 75: 026604 (2007). 

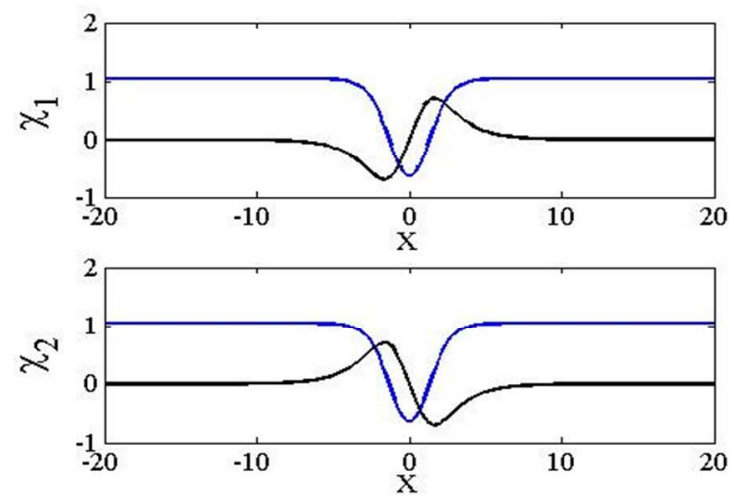

Fig. 1. Bound state Josephson vortices solution obtained numerically for the parameter values $\mu=1, \omega=1$ and $k=0.1$. The blue curves show the real parts and the black curves represent the imaginary parts of $\chi_{1}$ and $\chi_{2}$.

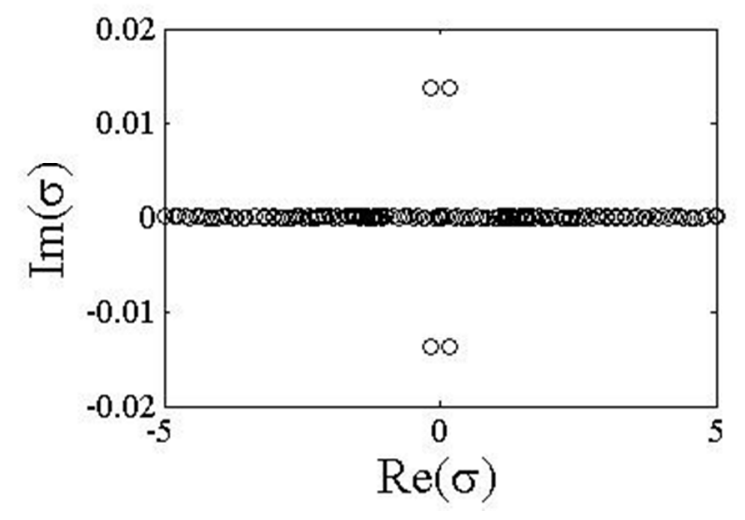

Fig. 2. The layout of eigenvalues for the Josephson vortices solution $\mu=1, \omega=1$ and $k=0.23$. Two pairs of eigenvalues are not on the horizontal axis and depicts the instability of the solution. 


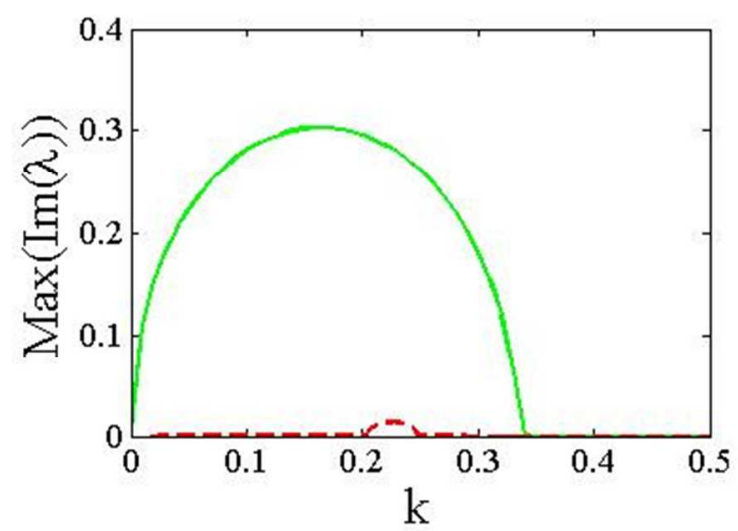

Fig.3. The graph of coupling strength $k$ versus the maximum value of the imaginary parts of the eigenvalues. The dotted curve is for bound state Josephson vortices solution while the solid curve is for coupled dark soliton solution.

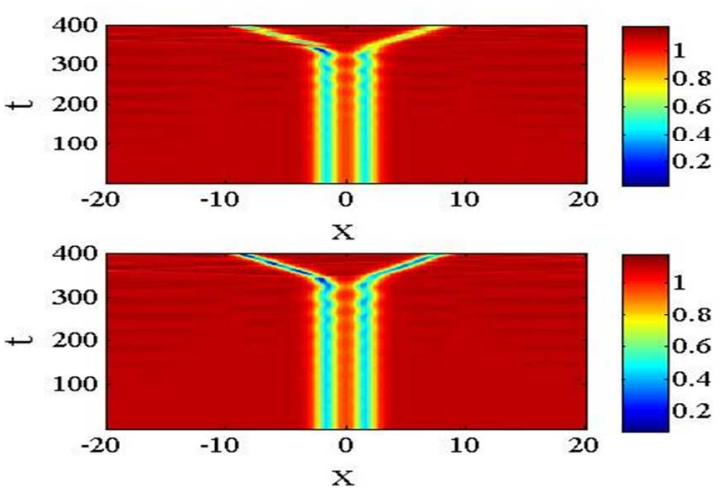

Fig.4. The contour plot for the time evolution of the bound state solution for $k=0.23, \mu=1, \omega=1$. Because of the instability of the solution, radiation are emerging and the solution moves away from the mean position. 